\title{
N91-22323
}

\section{COMBINED STRUCTURES-CONTROLS OPTIMIZATION OF LATTICE TRUSSES}

A. V. Balakrishnan

Research supported in part under NASA Task Assignment 49: NAS1-18585.

253 


\title{
COMBINED STRUCTURES-CONTROLS OPTIMIZATION OF LATTICE TRUSSES
}

\author{
A. V. Balakrishnan
}

\begin{abstract}
The purpose of this paper is to demonstrate concretely the role that distributed parameter models can play in CSI, in particular in combined structures-controls optimization problems of importance in preliminary design. Closed form solutions can be obtained for performance criteria such as rms attitude error, making possible analytical solutions of the optimization problem. This is in contrast to the need for numerical computer solution involving the inversion of large matrices in traditional finite element model use. Another advantage of the analytic solution is that it can provide much needed "insight" into phenomena that can otherwise be obscured - or difficult to discem from numerical computer results.

As a compromise in level of complexity between a toy laboratory model and a real space structure we have chosen the lattice truss used in the EPS (Earth Pointing Satellite). The optimization problem chosen is a generic one: of minimizing the structure mass subject to a specified stability margin and to a specified upper bound on the rms attitude error ("tip response"), using co-located controller and sensors. Standard FEM treating each bar as a truss element is used, while the continuum model is anisotropic Timoshenko beam model. Performance criteria are derived for either model, except that for the distributed parameter model we obtain explicit closed form solutions. Numerical results obtained by the two models show complete agreement. Based on the continuum model we obtain a solution to the problem of optimal placement of actuators to minimize mean square attitude error. A canonical optimization problem is examined and shown to be trivial, and even capable of analytical solution, using the continuum model performance criteria formulas in contrast to the complex computer solutions based on FEM or truncated modal models currently in vogue.
\end{abstract}




\section{Introduction}

The most voiced criticism against the use of continuum models for structures is that they are (a) impossible to derive for a realistic structure and (b) even if it could be done, calculations using the model are equally impossible. We shall show that both statements are false - at least in the CSI optimization problem - in particular in preliminary design.

In combined controls-structures optimization, the optimization is the least difficult the real challenge is to derive expressions for the chosen performance criteria in terms of the controls/structures parameters. We shall show that such formulas are much simpler when continuum models are used - moreover in many cases we can derive explicit closed form expressions in terms of elementary function which can actually trivialize the optimization problem. In particular the techinques of optimization need no longer dominate.

The purpose of this paper is to demonstrate concretely the role that distributed parameter models can play in CSI, in particular in combined structures-controls optimization problems of importance in preliminary design. Closed form solutions can be obtained for performance criteria such as rms attitude error, making possible analytical solutions of the optimization problem. This is in contrast to the need for numerical computer solution involving the inversion of large matrices in traditional finite element model use. Another advantage of the analytic solution is that it can provide much needed "insight" into phenomena that can otherwise be obscured - or difficult to discern from numerical computer results.

As a compromise in level of complexity between a toy laboratory model and a real space structure we have chosen the lattice truss used in the EPS (Earth Pointing Satellite). This is described in Section 1. The optimization problem chosen is a generic one: of minimizing the structure mass subject to a specified stability margin and to a specified upper bound on the rms attitude error ("tip response"). The mathematical statement of 255 
the performance criteria is given in Section 2. The first step is to evaluate the performance criterion for a given control configuration - we consider co-located sensor/controls only. The finite element model is described in Section 3, and the continuum model in Section 4. The dynamic state space model is seen to be identical in both cases except for state space dimension. Section 5 derives the performance criteria valid for either model, except that for the distributed parameter model we obtain explicit closed form solutions. Section 6 compares the numerical results obtained by the two models, showing complete agreement. As a byproduct of our analysis, we obtain a solution to the problem of optimal placement of actuators to minimize mean square attitude error - in Section 7. An optimization problem per se - a canonical one - is treated in Section 8 and by virtue of our explicit formulas for performance indices in terms of structure/control parameter, shown to be "trivial" and even capable of analytical solution - in contrast to computer solutions using FEM or truncated modal models as in $[9,10]$.

We should note that structural engineers (Noor, et al. [1, 2] and Renton [3]) have already voiced the advantages of continuum models in preliminary structure design what is new here is the application to control design, to the Controls-Structures Interaction problem. 


\section{The Physical Article}

The physical structure (Figure 1) is a lattice of rectangular bays, each single-laced single-bay. Offset at each end is an antenna. The controllers are force and moment actuators with co-located attitude as well as rate sensors stationed at arbitrary locations along the structure. Table 1 is a breakdown of the parameters describing each bay.

TABLE 1

Element Properties

\begin{tabular}{l|c|c|c|c}
\hline & Battens & $\begin{array}{c}\text { Longitudinal } \\
\text { Bars }\end{array}$ & $\begin{array}{c}\text { Diagonal } \\
\text { Bars }\end{array}$ & $\begin{array}{c}\text { Cross Bracing } \\
\text { in Battens }\end{array}$ \\
\hline \begin{tabular}{l} 
Length $L$ \\
\hline $\begin{array}{l}\text { Sectional } \\
\text { Area } A\end{array}$
\end{tabular} & $b$ & $\ell$ & $d$ & $\delta$ \\
\hline $\begin{array}{l}\text { Elastic } \\
\text { Modulus } E\end{array}$ & $A_{b}$ & $A_{\ell}$ & $A_{d}$ & $A_{\delta}$ \\
\hline \begin{tabular}{l} 
Mass Density $m$ \\
\hline $\begin{array}{l}\text { Element } \\
\text { Mass }=\rho A L\end{array}$
\end{tabular}$\rho_{b}$ & $\rho_{\ell}$ & $E_{d}$ & $E_{\delta}$ \\
\hline $\begin{array}{l}\text { Element } \\
\text { Stiffness }=E A / L\end{array}$ & $m_{b}$ & $m_{\ell}$ & $m_{d}$ & $\rho_{\delta}$ \\
\hline
\end{tabular}

The beam geometry is shown in Figure 2. By the "nominal" structure, we shall mean the following choice of structural parameters:

$$
\begin{gathered}
b=\ell ; \quad d=\delta=\sqrt{2} \ell \\
A_{b}=A_{\ell}=A_{d}=A_{\delta}=A \\
=E_{b}=E_{\ell}=E_{d}=E_{\delta}=E \\
=\rho_{b}=\rho_{\ell}=\rho_{d}=\rho_{\delta}=\rho \\
L=n \ell ; \\
257
\end{gathered}
$$


Nominal values:

$$
\begin{aligned}
& n=9 \\
& \ell=3 \mathrm{~m} \\
& \rho=3250 \\
& E=2.759 \times 10^{11} \\
& A=2.468 \times 10^{-4} \mathrm{~m}^{2} .
\end{aligned}
$$




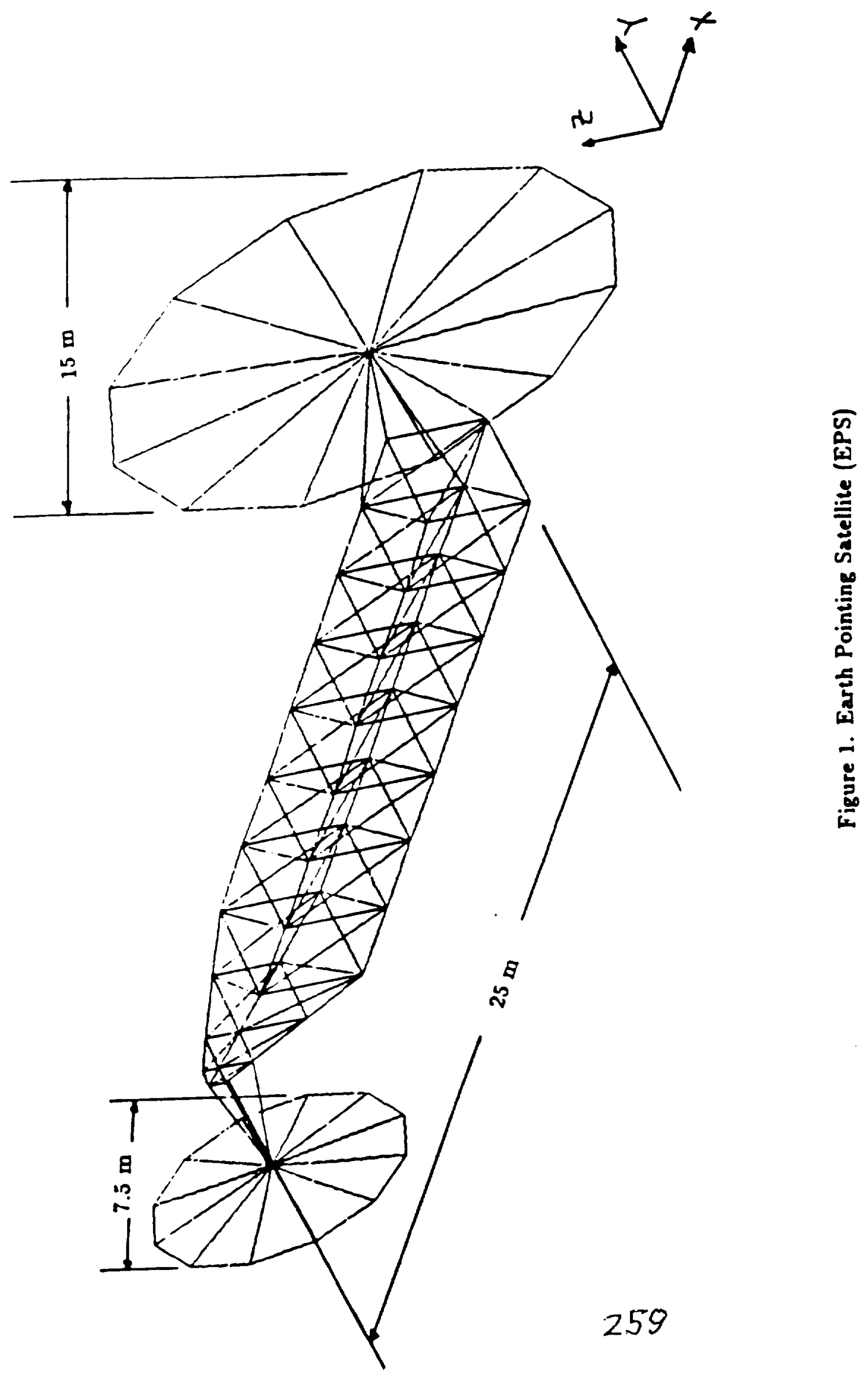




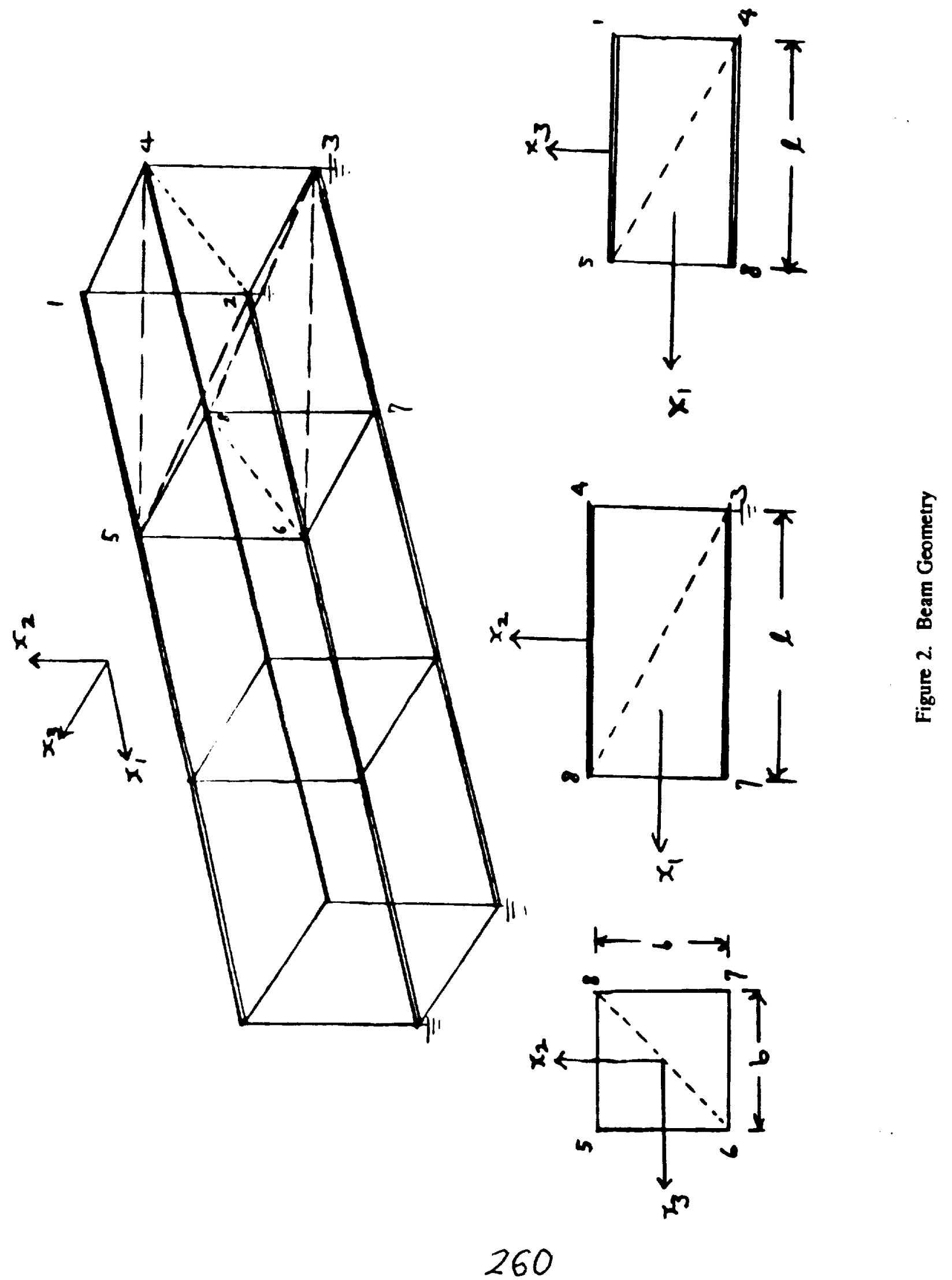




\section{Performance Criteria}

As in previous CIS optimization studies (see [9] and the references therein) the objective is to minimize the total mass of the structure, including the controller mass, subject to meeting specified performance requirements, which we shall now describe in mathematical terms.

The performance criteria chosen are:

(i) the mean-square attitude error due to sensor noise (using co-located sensors/ actuators). As we shall see, this actually depends on the steady state "tip" response to step inputs so that "noise" notions can be eschewed if necessary.

(ii) the "stability margin": defined as the sum of the absolute values of the real parts of the closed-loop eigenvalues. This is one measure of stability among very many (see, e.g., [10]). We choose this one because it is essentially equivalent to any other one but has the advantage that we can derive a simple closed-form expression for it.

Let us now define the criteria more precisely. First of all we assume the control law to be PD ("proportional plus derivative") as in classical servo design. Let $\mathrm{v}_{p}(t)$ denote the "displacement" or "attitude" vectors at the sensor locations and let $v_{r}(t)$ the rate. Then $U(t)$ the control is defined to be

$$
U(t)=\alpha v_{p}(t)+\gamma v_{r}(t)
$$

where $\alpha$ and $\gamma$ are (positive) scalar "gains." This is not altogether a "simplifying" assumption - that the scalar rate feedback is actually optimal is shown in [4]. The beam-axis being the $x_{1}$-axis, we have, with $L$ denoting the beam-length:

$$
0 \leq x_{1} \leq L \text {. }
$$

Let $f(0), f(L)$ denote the 6-DOF displacement vectors at the ends. Let

$$
f(0)=\left|\begin{array}{c}
u(0) \\
v(0) \\
w(0) \\
\phi_{1}(0) \\
\phi_{2}(0) \\
\phi_{3}(0)
\end{array}\right| ; \quad f(L)=\left|\begin{array}{c}
u(L) \\
v(L) \\
w(L) \\
\phi_{1}(L) \\
\phi_{2}(L) \\
\phi_{3}(L)
\end{array}\right|
$$


where $u, v, w$ are the 3-DOF displacements, $\phi_{1}$ the torsion angle about the beam axis, and $\phi_{2}, \phi_{3}$ about the mutually perpendicular axes. Then the mean square attitude error is defined by

$$
\begin{aligned}
\sigma_{a}^{2}=\overline{u(0)^{2}+\overline{u(L)^{2}}} & +\overline{v(0)^{2}+v(L)^{2}}+\overline{w(0)^{2}+w(L)^{2}} \\
& +\left|r_{0}\right|^{2} \overline{\phi_{1}(0)^{2}}+\left|r_{L}\right|^{2} \overline{\phi_{1}(L)^{2}}
\end{aligned}
$$

where the bars denote time averages, $|r|$ being the length of the moment arm as required.

Under our feedback law (often referred to as "positive-definite" feedback) the closedloop system is guaranteed to be stable. Assuming no damping in th structure (as we shall indeed do), the real parts of the closed-loop eigenvalues are guaranteed negative (see Section 5) - or if we assume the structure is already damped we have stability enhance ment corresponding to the increment, the real parts being now more negative. Let $\sigma_{i}$ denote the real part increment corresponding to the $i$ th closed-loop mode. Then the infinite series

$$
\sum_{i}^{\infty}-\sigma_{i}
$$

converges. Denote the sum by $\sigma_{s}$. We shall take this to be the "stability index" - the higher the index, the more stable. 


\section{The Finite Element Model}

Since most of the techniques in developing the FEM are standard, we shall only present the relevant numerical data. Each bar is taken as a truss element with 6 DOF. There are $(13+5)$ elements per bay, and hence $(13 \times 9+5)=122$ elements for 9 bays. There are 40 nodes with 3 DOF each, so that the stiffness matrix $A$ and the mass/inertia matrix $M$ are $120 \times 120$. The state vector is thus $120 \times 1$. The displacements along the axis of the truss are then expressed (or, rather, extrapolated):

$$
\begin{gathered}
u(k \ell)=u(k \ell, 0,0)=\frac{u(k \ell, 0-b / 2)+u(k \ell, 0+b / 2)+u(k \ell,-b / 2,0)+u(k \ell,+b / 2,0)}{4} \\
v(k \ell)=v(k \ell, 0,0)=\frac{v(k \ell, 0-b / 2)+v(k \ell, 0+b / 2)+v(k \ell,-b / 2,0)+v(k \ell,+b / 2,0)}{4} \\
w(k \ell)=w(k \ell, 0,0)=\frac{w(k \ell, 0-b / 2)+w(k \ell, 0+b / 2)+w(k \ell,-b / 2,0)+w(k \ell,+b / 2,0)}{4} \\
\phi_{1}(k \ell)=\frac{1}{2}\left[\frac{w(k \ell,+b / 2,0)-w(k \ell,-b / 2,0)}{b}-\frac{v(k \ell, 0,+b / 2)-v(k \ell, 0,-b / 2)}{b}\right] \\
\phi_{2}(k \ell)=\frac{u(k \ell, 0,+b / 2)-u(k \ell, 0,-b / 2)}{b} \\
\phi_{3}(k \ell)=\frac{u(k \ell,+b / 2,0)-u(k \ell,-b / 2,0)}{b}
\end{gathered}
$$

where $k$ is an integer and $0 \leq k \ell \leq L$. Allowing for $m$ controllers at $k=k_{1}, k_{2}, \ldots, k_{m}$, the corresponding relations can be represened by a $6 m \times 120$ matrix acting on the state vector. (We consider in this paper $m=1,2$ or 3 .) Let $B$ denote the transpose of this matrix. Then the state space dynamics with co-located sensors can be described by:

$$
M \ddot{x}+A x+B U+B N_{a}=0
$$

with sensor data:

$$
\begin{aligned}
& \mathrm{v}_{p}(t)=B^{*} x(t)+N_{p}(t) \\
& v_{r}(t)=B^{*} x(t)+N_{r}(t)
\end{aligned}
$$

where $U(\cdot)$ denotes the control; $N_{a}(\cdot), N_{p}(\cdot), N_{r}(\cdot)$ model additive noise taken as (mutually independent) white Gaussian with spectral density matrices $d_{a} I, d_{p} I, d_{r} I$ respectively, $I$ being the identity matrix. 


\section{The Continuum Model}

As we noted in Section 1, the problem of producing an "exact," "three-D" continuum model for a real-world structure like the truss we are considering can be a formidable one - although research in this area looks promising [5]. One way out of this difficulty is to exploit where possible the special nature of the truss - in our case it is a lattice of bays along the same axis numerous enough so that it is even visually "beam-like." In that case there are many ways to approximate by "one- $D$ " beams - without going into the details of this theory, suffice it to say that the approach by Noor and Russell [2] is the one adapted here. We thus create an "equivalent" (referring to [2] for the precise sense) onedimensional anisotropic Timoshenko beam as follows.

$u$ denoting axial (longitudinal) displacement (along the $x_{1}$-axis);

$\phi_{1}$ the torsion angle about this axis ,

$w, \phi_{2}$ denoting the transverse bending displacement in the $x_{1}-x_{3}$ plane and torsion angle about the $x_{2}$-axis,

$v, \phi_{3}$ denoting the transverse bending displacement in the $x_{1}-x_{2}$ plane and torsion angle about the $x_{3}$-axis,

the three axes being mutually perpendicular; $0 \leq x_{1} \leq L$, $L$ being the beam length $=n \ell ; n=$ number of bays

The Timoshenko equations (valid between control nodes) are:

$$
\begin{gathered}
m_{11} \ddot{u}-c_{11} u^{\prime \prime}-c_{14} \mathrm{v}^{\prime \prime}-c_{15} w^{\prime \prime}-c_{15} \phi_{2}^{\prime}+c_{14} \phi_{3}^{\prime}=0 \\
m_{22} \ddot{v}-c_{44} \mathrm{v}^{\prime \prime}-c_{14} u^{\prime \prime}+c_{44} \phi_{3}^{\prime}=0 \\
m_{33} \ddot{w}-c_{55} w^{\prime \prime}-c_{15} u^{\prime \prime}-c_{55} \phi_{2}^{\prime}=0 \\
m_{44} \ddot{\phi}_{1}-c_{66} \phi_{1}^{\prime \prime}-c_{36} \phi_{2}^{\prime \prime}-c_{26} \phi_{3}^{\prime \prime}=0 \\
m_{55} \ddot{\phi}_{2}+m_{56} \ddot{\phi}_{3}+c_{15} u^{\prime}+c_{55} w^{\prime}-c_{36} \phi_{1}^{\prime \prime}+c_{55} \phi_{2}-c_{33} \phi_{2}^{\prime \prime}-c_{23} \phi_{3}^{\prime \prime}=0 \\
m_{66} \ddot{\phi}_{3}+m_{56} \ddot{\phi}_{2}-c_{14} u^{\prime}-c_{44} \mathrm{v}^{\prime}-c_{26} \phi_{1}^{\prime \prime}-c_{23} \phi_{2}^{\prime \prime}+c_{44} \phi_{3}-c_{22} \phi_{3}^{\prime \prime}=0
\end{gathered}
$$

where the superdots denote time-derivatives and the primes, space derivatives. The coeffi- 
cients of these dynamic equations are related to the truss parameters as follows: (cf. [2]): The mass coefficients are given by:

$$
\begin{aligned}
m_{11}=m_{22}=m_{33}=\frac{4 m_{b}+4 m_{\ell}+4 m_{d}+m_{\delta}}{\ell} \\
m_{44}=2 m_{55}=2 m_{66}=\frac{\ell\left(8 m_{b}+12 m_{\ell}+8 m_{d}+m_{\delta}\right)}{6 \mu^{2}} \\
m_{56}=\frac{\ell m_{\delta}}{12 \mu^{2}}
\end{aligned}
$$

The stiffness (flexibility) $c_{i j}$ are given by:

$$
\begin{aligned}
& c_{11}=4 \ell S_{\ell}+\frac{4 \ell S_{b} S_{d} \mu^{2}}{S_{d}+S_{b}\left(\ell+\mu^{2}\right)} \\
& c_{44}=\frac{c_{14}}{\mu}=c_{55}=\frac{c_{15}}{\mu}=\frac{2 \ell S_{b} S_{d}}{S_{d}+S_{b}\left(\ell+\mu^{2}\right)} \\
& c_{22}=c_{33}=\frac{\ell^{3} S_{\ell}}{\mu^{2}}+\frac{\ell^{3} S_{b} S_{d}}{4\left(S_{d}+S_{b}\left(\ell+\mu^{2}\right)\right)} \\
& c_{23}=\frac{\ell^{3} S_{b} S_{d}}{4\left(S_{d}+S_{b}\left(\ell+\mu^{2}\right)\right)} \\
& c_{66}=-2 c_{26}=-2 c_{36}=\frac{\ell^{3} S_{b} S_{d}}{\mu^{2}\left(S_{d}+S_{b}\left(\ell+\mu^{2}\right)\right)}
\end{aligned}
$$

where

$$
\mu=\frac{\ell}{b} \text {. }
$$

In order not to complicate matters unduly in this demonstration, we shall freeze all parameters except the cross-sectional area $A$ which will then be the structural parameter to be optimized. In this case

$$
\begin{gathered}
c_{11}=\frac{(40+24 \sqrt{2}) E A}{9+4 \sqrt{2}} \\
c_{14}=-c_{15}=c_{44}=c_{55}=\frac{2 E A}{1+2 \sqrt{2}} \\
c_{22}=c_{33}=\frac{(2725+1476 \sqrt{2}) E A \ell^{2}}{2628+1336 \sqrt{2}} \quad \text { (Newton) } \\
265
\end{gathered}
$$




$$
\begin{array}{ccc}
c_{23} & =-\left(\frac{(97+140 \sqrt{2}) E A \ell^{2}}{2628+1336 \sqrt{2}}\right) \quad \text { (Newton) } \mathrm{m}^{2} \\
c_{26}=-c_{36}= & \frac{1}{2} c_{66}=\frac{(16+33 \sqrt{2}) E A \ell^{2}}{296+130 \sqrt{2}} & (\text { Newton}) \mathrm{m}^{2} \\
m_{11}= & m_{22}=m_{33}=(8+5 \sqrt{2}) A \rho \quad \mathrm{kg} / \mathrm{m} & \\
m_{44}= & 2 m_{55}=2 m_{66}=\frac{(20+9 \sqrt{2}) A \ell^{2} \rho}{6} \quad \mathrm{~kg} \cdot \mathrm{m} \\
m_{56} & =-\frac{\left(A \ell^{2} \rho\right)}{6 \sqrt{2}} \quad \mathrm{~kg} \cdot \mathrm{m} .
\end{array}
$$

Once the coefficients $c_{i j}, m_{i j}$ are defined (on whatever basis), we can develop the generic state space dynamic model analogous to the FEM formulas (3.1), (3.2), (3.3):

$$
\left.\begin{array}{l}
M \ddot{x}+A x+B u+B N_{a}=0 \\
v_{p}(t)=B^{*} x(t)+N_{p}(t) \\
\mathrm{v}_{r}(t)=B^{*} \dot{x}(t)+N_{a}(t)
\end{array}\right\}
$$

where $N_{a}(\cdot), N_{p}(\cdot), N_{r}(\cdot)$ are white Gaussian noise with spectral density $d_{a} I, d_{p} I, d_{f} I$ respectively. ${ }^{\dagger}$ Only, the dimension of the state $x(t)$ is not finite. The technique of derivation is also different, in particular in the role of the energy. See $[6,7]$ for details. Here we can only summarize the basic results.

\section{Case 1: One Controller}

We begin with constructing the state space model for one controller ("midcontroller"), and offset masses at each end, referring to [4, 6] again for more details and to [7] where the general case of distributed control is treated. Thus the state $x(t)$ at time $t$ is defined by

$$
x(t)=\left|\begin{array}{l}
f(\cdot, t) \\
f(0, t) \\
f\left(s_{2}, t\right) \\
f(L, t)
\end{array}\right|
$$

† See [7] for generalization to arbitrary diagonal matrices. 
where $s$ parametrizes the beam axis, zero denoting one end and $L$ the other, $L$ being the total beam length, and $s_{2}$ denotes the location of the mid-controllers and $f(\cdot, t)$ denotes a (6×1 vector) function of $s, 0 \leq s \leq L$, representing displacements and angles:

$$
f(s, t)=\left|\begin{array}{l}
u(s, t) \\
\mathrm{v}(s, t) \\
w(s, t) \\
\phi_{1}(s, t) \\
\phi_{2}(s, t) \\
\phi_{3}(s, t)
\end{array}\right|
$$

The stiffness operator $A$ is defined as follows:

$$
\begin{aligned}
& A x=\left|\begin{array}{l}
g \\
\mathrm{v}
\end{array}\right| ; \quad x=\left|\begin{array}{c}
f(\cdot) \\
f(0) \\
f(s) \\
f(L)
\end{array}\right| \\
& g(s)=-A_{2} f^{\prime \prime}(s)+A_{1} f^{\prime}(s)+A_{0} f(s), \quad 0<s<s_{2} ; s_{2}<s<L .
\end{aligned}
$$

The derivative $f^{\prime}(\cdot)$ has a discontinuity at $s=s_{2}$, and

$$
v=\left|\begin{array}{c}
-L_{1} f(0)-A_{2} f(0) \\
-A_{2}\left(f^{\prime}\left(s_{2}+\right)-f^{\prime}\left(s_{2}-\right)\right) \\
L_{1} f(L)+A_{2} f^{\prime}(L)
\end{array}\right|
$$

and thus defined, the potential energy

$$
=[A x, x]=\int_{0}^{L}\left[H\left|\begin{array}{l}
f^{\prime}(s) \\
f(s)
\end{array}\right|,\left|\begin{array}{l}
f^{\prime}(s) \\
f(s)
\end{array}\right|\right] d s \geq 0
$$

where

$$
\begin{gathered}
H=\left|\begin{array}{cccc}
C_{1} & 0 & 0 & -C_{2} \\
0 & C_{3} & 0 & 0 \\
0 & 0 & A_{0} \\
-C_{2}^{*} & 0
\end{array}\right| \\
A_{2}=\left|\begin{array}{cc}
C_{1} & 0 \\
0 & C_{3}
\end{array}\right| \\
267
\end{gathered}
$$




$$
\begin{aligned}
& C_{1}=\left|\begin{array}{ccc}
c_{11} & c_{14} & c_{15} \\
c_{14} & c_{44} & 0 \\
c_{15} & 0 & c_{55}
\end{array}\right| \\
& C_{3}=\left|\begin{array}{ccc}
c_{66} & c_{36} & c_{26} \\
c_{36} & c_{33} & c_{23} \\
c_{26} & c_{23} & c_{22}
\end{array}\right| \\
& A_{1}=\left|\begin{array}{cc}
0 & C_{2} \\
-C_{2}^{*} & 0
\end{array}\right| \\
& C_{2}=\left|\begin{array}{ccc}
0 & -c_{15} & c_{14} \\
0 & 0 & c_{44} \\
0 & -c_{55} & 0
\end{array}\right| \\
& \left.A_{0}=\text { Diag. [0, } 0,0,0, c_{55}, c_{44}\right] \\
& L_{1}=\left|\begin{array}{cc}
0 & -C_{2} \\
0 & 0
\end{array}\right| .
\end{aligned}
$$

$B$ is defined by

$$
B U=\left|\begin{array}{c}
0 \\
0 \\
U \\
0
\end{array}\right|, \quad U \text { is } 6 \times 1
$$

and

$$
B^{*} x=f\left(s_{2}\right) \quad(6 \times 1) .
$$

The mass/inertia operator $M$ is defined

$$
M x=\left|\begin{array}{l}
M_{0} f(\cdot) \\
M_{b, 0} f(0) \\
M_{c} f\left(s_{2}\right) \\
M_{b, L} f(L)
\end{array}\right|
$$


where

$$
M_{0}=\left|\begin{array}{cccccc}
m_{11} & \cdot & \cdot & \cdot & \cdot & \cdot \\
\cdot & m_{22} & \cdot & \cdot & \cdot & \cdot \\
\cdot & \cdot & m_{33} & \cdot & \cdot & \cdot \\
\cdot & \cdot & \cdot & m_{44} & \cdot & \cdot \\
\cdot & \cdot & \cdot & \cdot & m_{55} & m_{56} \\
\cdot & \cdot & \cdot & \cdot & m_{56} & m_{66}
\end{array}\right|
$$

Diag. $M_{b_{0}}=\left(m_{0}, m_{0}, m_{0}\right.$, Diag. $\left.I_{0}\right)$

where $m_{0}$ is the offset mass at $s=0$ and $I_{0}$ is its moment of inertia about zero, and similarly

$$
\text { Diag. } M_{b_{L}}=\left(m_{L}, m_{L}, m_{L}, \text { Diag. } I_{L}\right) \text {. }
$$

See also [6] for more on $M_{b_{0}}, M_{b_{L}}$.

$$
M_{c}=\left|\begin{array}{llllll}
m_{c} & 0 & 0 & 0 & 0 & 0 \\
0 & m_{c} & 0 & 0 & 0 & 0 \\
0 & 0 & m_{c} & 0 & 0 & 0 \\
0 & 0 & 0 & & & \\
0 & 0 & 0 & & I_{c} & \\
0 & 0 & 0 & & &
\end{array}\right|
$$

where $m_{c}$ is the force actuator (rotor) mass and $I_{c}$ the moment of inertia of the moment actuator about its center of gravity. The m.s. attitude error matrix is defined by

$$
\lim _{T \rightarrow \infty}\left\{\frac{1}{T} \int_{0}^{T} f(0, t) f(0,)^{*} d t+\frac{1}{T} \int_{0}^{T} f(L, t) f(L, t)^{*} d t\right\}
$$

and $\sigma_{a}^{2}$ is the sum of the diagonal terms as defined.

\section{Case 2: Two Controllers}

Next we consider the case of two controllers, one at each end. Here, since there is no mid-controller, we may delete that entry in the state. Thus

$$
x(t)=\left|\begin{array}{l}
f(\cdot, t) \\
f(0, t) \\
f(L, t)
\end{array}\right|
$$




$$
M x=\left|\begin{array}{r}
M_{0} f(\cdot, t) \\
M_{b, 0} f(0, t) \\
M_{b, L} f(L, t)
\end{array}\right| .
$$

where the end-masses $m_{0}, m_{L}$ must now include the actuator moving masses, and similarly for the moment of inertia matrices. We shall use the notation

$$
M_{c}=\left|\begin{array}{cc}
M_{b, 0} & 0 \\
0 & M_{b, L}
\end{array}\right|
$$

With $U(\cdot)$ denoting the control vector, $(12 \times 1)$, we have

$$
\begin{gathered}
B U=\left|\begin{array}{c}
0 \\
U
\end{array}\right| \\
B^{*} x=\left|\begin{array}{c}
f(0, t) \\
f(L, t)
\end{array}\right| .
\end{gathered}
$$

Finally

$$
\begin{gathered}
A x=\left|\begin{array}{l}
g \\
\mathrm{v}
\end{array}\right| \\
\mathrm{v}=\left|\begin{array}{r}
-L_{1} f(0)-A_{2} f^{\prime}(0) \\
L_{1} f(L)+A_{2} f^{\prime}(L)
\end{array}\right| \\
g(s)=-A_{2} f^{\prime \prime}(s)+A_{1} f^{\prime}(s)+A_{0} f(s), \quad 0<s<L .
\end{gathered}
$$

Here the mean square attitude error-matrix

$$
=\lim _{T \rightarrow \infty} \frac{1}{T} \int_{0}^{T}\left(B^{*} x(t)\right)\left(B^{*} x(t)\right)^{*} d t
$$

\section{Case 3: Three Controllers}

In this case we have a mid-controller at $s=s_{2}$ as well as a controller at each end. Here

$$
x(t)=\left|\begin{array}{l}
f(\cdot, t) \\
f(0, t) \\
f\left(s_{2}, t\right) \\
f(L, t)
\end{array}\right|,
$$


in other words the same state vector as in Case 1.

$$
M x(t)=\left|\begin{array}{r}
M_{0} f(\cdot, t) \\
M_{b, 0} f(0, t) \\
M_{b, 2} f\left(s_{2}, t\right) \\
M_{b, L} f(L, t)
\end{array}\right|
$$

where

$$
M_{0,2}=\left|\begin{array}{cccccc}
m_{c} & 0 & 0 & 0 & 0 & 0 \\
0 & m_{c} & 0 & 0 & 0 & 0 \\
0 & 0 & m_{c} & 0 & 0 & 0 \\
0 & 0 & 0 & & & \\
0 & 0 & 0 & & I_{c} & \\
0 & 0 & 0 & & &
\end{array}\right|
$$

where $m_{c}$ is the force actuator moving mass and $I_{c}$ is the moment of inertia about its center of gravity, corresponding to the "mid-controller."

$$
\begin{aligned}
& B U=\left|\begin{array}{c}
0 \\
U
\end{array}\right| \\
& B^{*} x=\left|\begin{array}{l}
f(0, t) \\
f\left(s_{2}, t\right) \\
f(L, t)
\end{array}\right| .
\end{aligned}
$$

We can calculate $\sigma_{a}^{2}$ from the diagonal terms of the mean square error matrix:

$$
\text { Diag. } \lim _{T \rightarrow \infty} \frac{1}{T} \int_{0}^{T}\left(B^{*} x(t)\right)\left(B^{*} x(t)\right)^{*} d t .
$$

which now has 18 entries. Once again we adopt the notation:

$$
M_{c}=\left|\begin{array}{cccc}
M_{0} & \cdot & \cdot & \cdot \\
\cdot & M_{b, 0} & \cdot & \cdot \\
\cdot & \cdot & M_{h 2} & \cdot \\
\cdot & \cdot & \cdot & M_{b L}
\end{array}\right|
$$




\section{Formulas for Performance Criteria}

We shall now develop formulas for the Performance Critera. First the mean square attitude error: Using either model FEM (3.1)-(3.3) or Continuum (4.1)-(4.2) we have the state space form:

$$
\begin{gathered}
M \ddot{x}+A x+B U+B N_{a}=0 \\
\mathrm{v}_{p}=B^{*} x+N_{p} \\
\mathrm{v}_{r}=B^{*} \dot{x}+N_{r}
\end{gathered}
$$

and

$$
U(t)=\alpha v_{p}(t)+\gamma v_{r}(t)
$$

Substituting this control law into the state equations we have:

$$
M \ddot{x}+\left(A+\alpha B B^{*}\right) x+\gamma B B^{*} \dot{x}+B\left(N_{a}+\alpha N_{p}+\gamma N_{r}\right)=0
$$

The steady-state output covariance matrix

$$
R_{a}=E\left(\left(B^{*} x(t)\right)\left(B^{*} x(t)\right)^{*}\right)=\left(\frac{\alpha^{2} d_{p}+d_{a}+\gamma^{2} d_{r}}{2 \gamma}\right)\left(B^{*}\left(A+\alpha B B^{*}\right)^{-1} B\right)
$$

where the matrix part:

$$
B^{*}\left(A+\alpha B B^{*}\right)^{-1} B
$$

is recognized as the steady-state input-output response matrix: it is the value at $\omega=0$ of the input-output transfer-function:

$$
B^{*}\left((i \omega)^{2} M+A+\alpha B B^{*}+(i \omega) \gamma B B^{*}\right)^{-1} B
$$

The scalar factor

$$
d=\frac{\alpha^{2} d_{p}+d_{a}+\gamma^{2} d_{r}}{2 \gamma}
$$

consolidates the "noise" part. For the case of two controllers the mean square attitude error $\sigma_{a}^{2}$ can be calculated from

\section{Diag. $\mathbf{R}_{a}$.}

For the third case of three controllers it is given by 


\section{Diag. $L R_{a} L^{*}$}

where

$$
L U=\left|\begin{array}{l}
f(0) \\
f(L)
\end{array}\right| .
$$

For Case 1 with one controller only, we have to calculate

$$
\begin{aligned}
& E\left(f(0, t) f(0, t)^{*}\right) \\
& E\left(f(L, t) f(L, t)^{*}\right) .
\end{aligned}
$$

Separately, expressing each as a transformation of the state:

$$
\begin{aligned}
& L_{1} x=f(0) \\
& L_{2} x=f(L) .
\end{aligned}
$$

In the FEM version, we have thus to invert the matrix

$$
\left(A+\alpha B B^{*}\right)
$$

which in our case is $120 \times 120$ - and of course can be done only numerically. For the continum model however we have to invent the operator

$$
\left(A+\alpha B B^{*}\right)^{-1}
$$

but - and this is the main point of departure - this can be done analytically. Referring to [7] for details, here we shall simply enumerate the formulas below.

\section{Case 1: One Controller Only}

$$
\begin{gathered}
E\left[f(0, t) f(0, t)^{*}\right]=\text { Diag. }\left(\frac{1}{\alpha}, \frac{1+s_{2}^{2}}{\alpha}, \frac{1+s_{2}^{2}}{\alpha}, \frac{1}{\alpha}, \frac{1}{\alpha}, \frac{1}{\alpha}\right) d \\
E\left[f(L, t) f(L, t)^{*}\right]=\text { Diag. }\left(\frac{1}{\alpha}, \frac{1+\left(L-s_{2}\right)^{2}}{\alpha}, \frac{1+\left(L-s_{2}\right)^{2}}{\alpha}, \frac{1}{\alpha}, \frac{1}{\alpha}, \frac{1}{\alpha}\right) d
\end{gathered}
$$

(each is a diagonal matrix!). Steady state step response corresponding to step input $U$ : 


$$
\begin{aligned}
& f(0, \infty)=\frac{1}{\alpha}\left|\begin{array}{cccc}
I_{3} & 0 & 0 & 0 \\
& 0 & 0 & -s_{2} \\
& 0 & s_{2} & 0 \\
0 & & I_{3} &
\end{array}\right| U \\
& f(L, \infty)=\frac{1}{\alpha}\left|\begin{array}{cccc}
I_{3} & 0 & 0 & 0 \\
& 0 & 0 & L-s_{2} \\
& 0 & s_{2}-L & 0 \\
0 & & I_{3} &
\end{array}\right| U
\end{aligned}
$$

Note: Controller at $s=s_{2}$.

\section{Case 2: Two End Controllers}

Diag. $E\left[f(0, t) f(0, t)^{*}\right]=$ Diag. $E\left[f(L, t) f(L, t)^{*}\right]$.

And the first four diagonal terms in either matrix are given in order by:

$$
\begin{aligned}
& =\left[1+\frac{\mu \beta}{\lambda \beta-2}\right]\left(\frac{1}{2 \alpha}\right) d \\
& =\left[1+\frac{\mu(\lambda \beta-1)}{\beta(\lambda \beta-2)}\right]\left(\frac{1}{2 \alpha}\right) d \\
& =\left[1+\frac{\mu(\lambda \beta-1)}{\beta(\lambda \beta-2)}\right]\left(\frac{1}{2 \alpha}\right) d \\
& =\left[\frac{1}{2 \alpha}+\frac{L}{4} \frac{c_{33}+\frac{\alpha L}{2}+\frac{c_{66}}{4}}{\left(c_{33}+\frac{\alpha L}{2}+\frac{c_{66}}{4}\right)\left(c_{66}+\frac{\alpha L}{2}\right)-\frac{c_{66}^{2}}{2}}\right] d
\end{aligned}
$$

where

$$
\begin{aligned}
& \mu=\frac{\alpha L}{2 c \delta}+\frac{L^{2}}{4} \\
& \beta=\mu+1 \\
& \lambda=\mu+2+\frac{\left(c_{11}-2 c\right)(2 \alpha+c \delta L)}{2 \alpha c \delta}
\end{aligned}
$$




$$
\begin{aligned}
& c=c_{44} \\
& \delta=\frac{1}{1+\frac{L^{2} \gamma}{12}} \\
& \gamma=\frac{c_{44}}{c_{33}-\frac{c_{66}}{4}} .
\end{aligned}
$$

\section{Case 3: Three Controllers}

Here we have to express the answers in terms of $6 \times 6$ matrices. Thus let

$$
\begin{aligned}
& D_{11}=\frac{E\left[f(0, t) f(0, t)^{*}\right]}{d} \\
& D_{22}=\frac{E\left[f\left(s_{2}, t\right) f\left(s_{2}, t\right)^{*}\right]}{d} \\
& D_{33}=\frac{E\left[f(L, t) f(L, t)^{*}\right]}{d} .
\end{aligned}
$$

Then

$$
\begin{aligned}
D_{11}= & d_{11}\left(s_{2}\right)-d_{12}\left(s_{2}\right)\left(d_{22}\left(s_{2}\right)+\left(d_{11}\left(L-s_{2}\right)^{-1}-\alpha\right)^{-1}\right)^{-1} d_{12}^{*}\left(s_{2}\right) \\
D_{22}= & \left(d_{22}^{-1}\left(s_{2}\right)+d_{11}\left(L-s_{2}\right)^{-1}-\alpha\right)^{-1} \\
D_{33}=\left(\alpha I+m_{3}\left(L-s_{2}\right)\right)^{-1}\left\{\alpha I+m_{3}\left(L-s_{2}\right)+m_{21}\left(L-s_{2}\right)\left(D_{22}\right) m_{21}^{*}\left(L-s_{2}\right)\right\} & \times\left(\alpha I+m_{3}\left(L-s_{2}\right)\right)^{-1}
\end{aligned}
$$

where

$$
\begin{aligned}
d_{11}(s)= & \left(\left(\alpha+m_{1}(s)\right)-m_{21}(s)^{*}\left(\alpha+m_{3}(s)\right)^{-1} m_{21}(s)\right)^{-1} \\
d_{22}(s)= & \left(\left(\alpha+m_{3}(s)\right)-m_{21}(s)\left(\alpha+m_{1}(s)\right)^{-1} m_{21}^{*}(s)\right)^{-1} \\
d_{12}(s)=-d_{11}(s) m_{21}(s)^{*}\left(\alpha I+m_{3}(s)\right)^{-1} & \\
& m_{1}(s)=\left|\begin{array}{cc}
\frac{c \delta(s)}{s} A_{11} & \frac{c \delta(s)}{2} A_{12} \\
\frac{c \delta(s)}{2} A_{12}^{*} & \frac{C_{3}}{s}+\frac{c s \delta(s)}{4} A_{22}
\end{array}\right| \\
& m_{3}(s)=\left|\begin{array}{cc}
\frac{c \delta(s)}{s} A_{11} & -\frac{c \delta(s)}{2} A_{12} \\
-\frac{c \delta(s)}{2} A_{12}^{*} & \frac{C_{3}}{s}+\frac{c s \delta(s)}{4} A_{22}
\end{array}\right|
\end{aligned}
$$




$$
m_{21}(s)=\left|\begin{array}{cr}
-\frac{c \delta(s)}{s} A_{11} & -\frac{c \delta(s)}{2} A_{12} \\
\frac{c \delta(s)}{2} A_{12}^{*} & -\frac{C_{3}}{s}+\frac{c s \delta(s)}{4} A_{22}
\end{array}\right|
$$

where

$$
\begin{gathered}
\delta(s)=\frac{1}{1+\frac{\gamma s^{2}}{12}} \\
A_{11}=\left|\begin{array}{ccc}
\frac{1}{\delta(s)}\left(\frac{c_{11}}{c}-2(1-\delta(s))\right. & 1 & -1 \\
1 & 1 & 0 \\
-1 & 0 & 1
\end{array}\right| \\
A_{12}=\left|\begin{array}{ccc}
0 & 1 & 1 \\
0 & 0 & 1 \\
0 & -1 & 0
\end{array}\right| \\
A_{22}=\left|\begin{array}{ccc}
0 & 0 & 0 \\
0 & 1 & 0 \\
0 & 0 & 1
\end{array}\right|
\end{gathered}
$$

\section{Stability Margin Formula}

From (5.5) it is clear that the closed-loop eigenfunctions are specified by

$$
\lambda^{2} M \phi+\left(A+\alpha B B^{*}\right) \phi+\gamma \lambda B B^{*} \phi=0
$$

(again, irrespective of whether we are using the FEM version (3.1)-(3.3) or the continuum version (4.1), (4.2)) where $\phi$ denotes the eigenvector ("mode shape"), with the eigenvalue $\lambda$ specified by

$$
\lambda^{2}[M \phi, \phi]+\left[\left(A+\alpha B B^{*}\right) \phi, \phi\right]+\lambda \gamma\left[B^{*} \phi, B^{*} \phi\right]=0
$$

where we may normalize the mode shape $\phi$ so that $[M \phi, \phi]=1$. Since $\alpha \geq 0$ and $\gamma \geq 0$, 
it follows that the eigenvalue $\lambda$ is given by:

$$
\lambda=-\sigma \pm i \omega
$$

where

$$
\begin{aligned}
\sigma & =-\gamma \frac{\left\|B^{*} \phi\right\|^{2}}{2} \\
\omega^{2} & =\omega_{0}^{2}-\sigma^{2} \\
\omega_{0}^{2} & =\left[\left(A+\alpha B B^{*}\right) \phi, \phi\right] .
\end{aligned}
$$

Thus the closed-loop eigenvalues have strictly negative real parts.

It is shown in [8] that the sum of the absolute values of the closed-loop eigenvalues is given by

$$
\gamma \operatorname{Tr} . M_{c}^{-1}
$$

in all cases, where in $M_{c}$ only moving parts of the actuators must be considered (as opposed to the stationary mass such as the armature mass). The simplicity of this formula is striking when compared with taking the sum of the inverses of the absolute values of the real parts of the closed loop eigenvalues for a finite number of modes as in [10]. We may note that for zero natural damping (or with damping, if we consider only the increment),

$$
\sum_{1}^{\infty} \frac{1}{\left|\sigma_{i}\right|}=\infty
$$

for any continuum model. Again, (5.8) applies for the FEM version (3.1)-(3.3), as well as for the continuum model (4.1), (4.2). 


\section{Numerical Results}

In this section we compare the numerical results by the two methods for the mean square attitude errors (and equivalently, tip response), for each of the three cases: one, two and three controllers - using the nominal values (cf. Section 1) for the truss parameters.

\section{Case 1: One Controller}

\section{FEM}

The tip response $f(0, \infty)$ and $f(L, \infty)$ was calculated for:

$$
\begin{array}{ll}
\alpha=c_{44}=35571851.2 ; & s_{2}=0, \frac{L}{9}, \frac{L}{3}, \frac{L}{2}, \\
\alpha=10,000 ; & s_{2}=0, \frac{L}{9}, \frac{L}{3}, \frac{L}{2}, \\
\alpha=100 ; & s_{2}=0, \frac{L}{9}, \frac{L}{3}, \frac{L}{2},
\end{array}
$$

both $f(0, \infty)$ and $f(L, \infty)$ values were exactly the same as the values predicted by the continuum model and hence are not displayed.

\section{Case 2: Two Controllers}

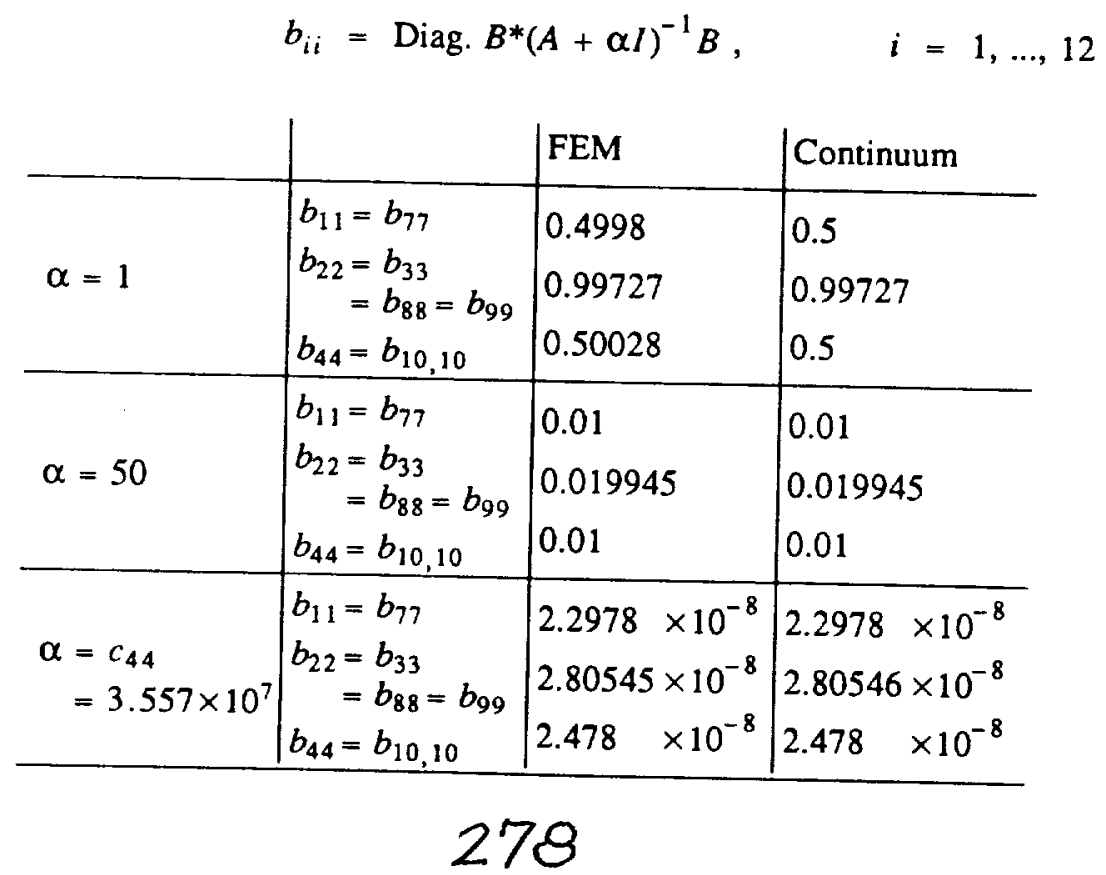




\section{Case 3: Three Controllers}

$$
\begin{aligned}
\alpha & =3.557 \times 10^{7} \\
b_{i i} & =i=1, \ldots, 18
\end{aligned}
$$

\begin{tabular}{l|l|l}
\hline \multirow{2}{*}{$\begin{array}{l}\text { Actuator } \\
\text { Position }\end{array}$} & $b_{11} \times 10^{8}$ & $b_{11} \times 10^{8}$ \\
\cline { 2 - 3 } & FEM & Continuum \\
\hline$s=\frac{L}{9}$ & 1.5119 & 1.5127 \\
\hline$s=\frac{3 L}{9}$ & 1.8366 & 1.8370 \\
\hline$s=\frac{4 L}{9}$ & 1.9461 & 1.9465 \\
\hline$s=\frac{5 L}{9}$ & 2.0330 & 2.0333 \\
\hline$s=\frac{6 L}{9}$ & 2.1031 & 2.1033 \\
\hline$s=\frac{7 L}{9}$ & 2.1602 & 2.1603 \\
\hline
\end{tabular}

In other words the FEM and the continuum gave exactly the same numerical results within (the SUN-386i) computer accuracy in all cases. 
SUM OF M.S. AXIAI DISPLACEMENTS AT BOTH ENDS

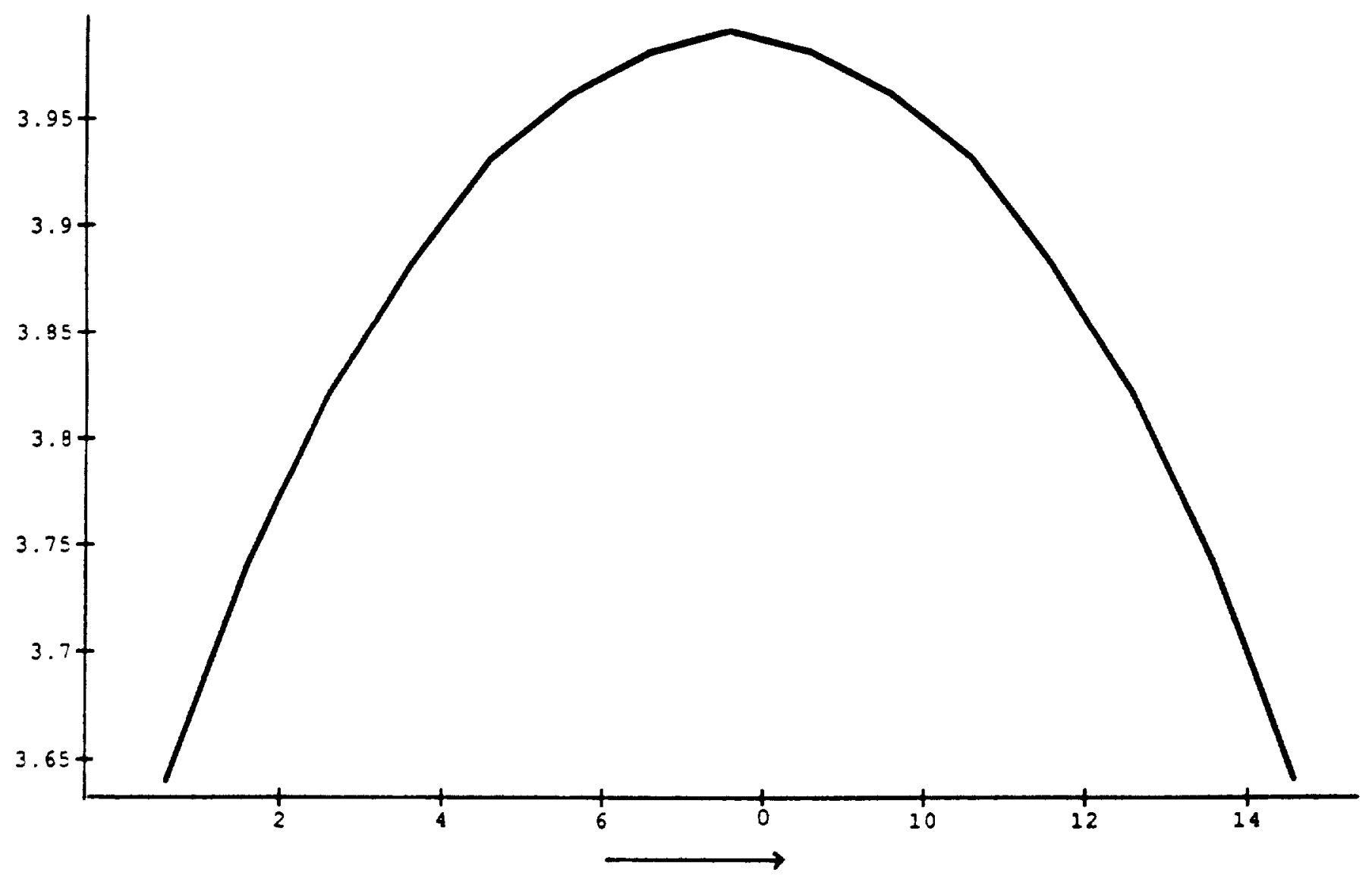

FIGURE 3

MIDACTUATOR POSITION: INCREMENTS L/16 
SUM OF M.S. BENDING DISPLACEMENTS AT BOTH ENDS

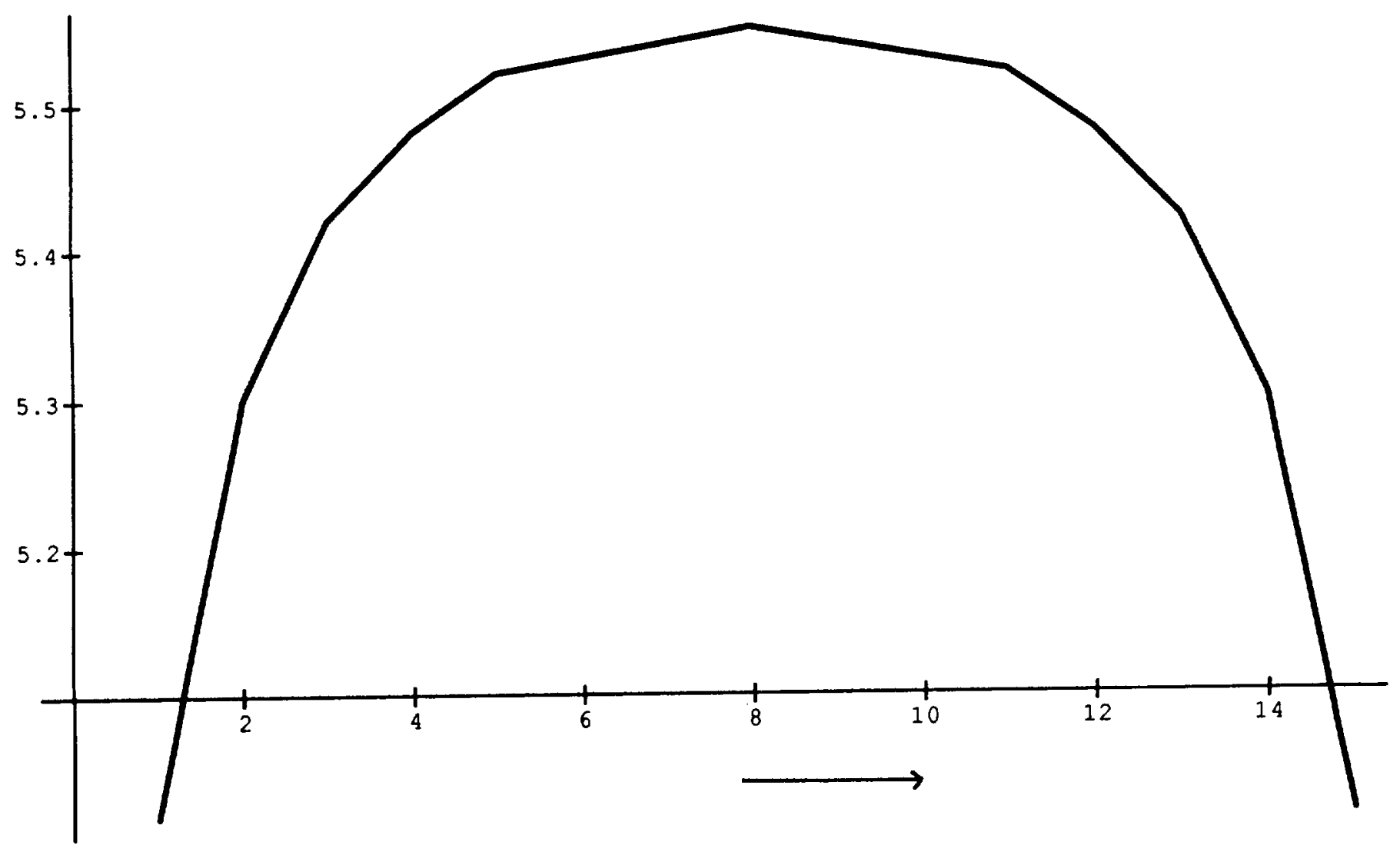

FIGURE 4

MIDACTUATOR POSITION: INCREMENTS L/16 
SUM OF M.S. TORSION AT BOTH ENDS

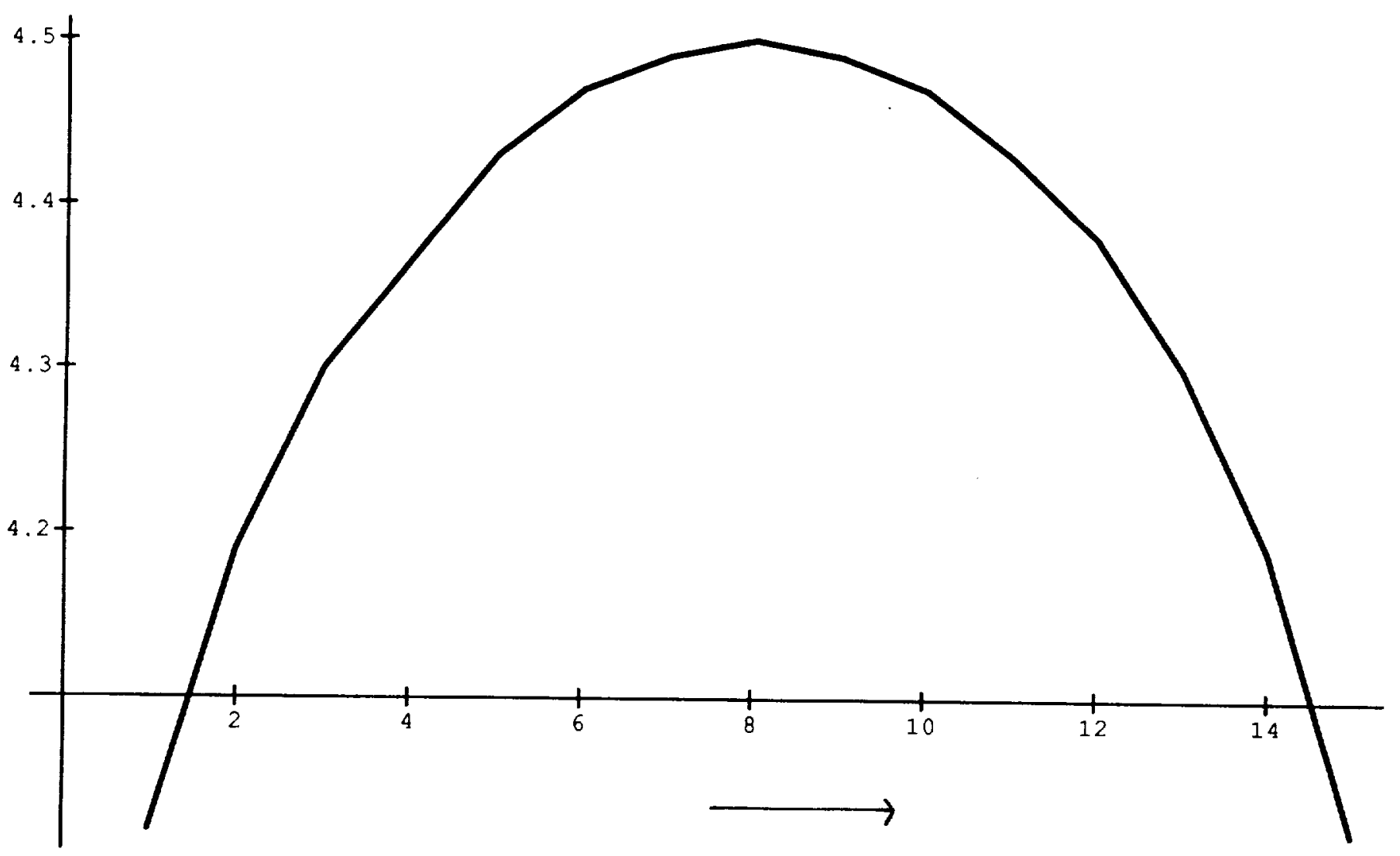

FIGURE 5

MIDACTUATOR POSITION: INCREMENTS L/16 


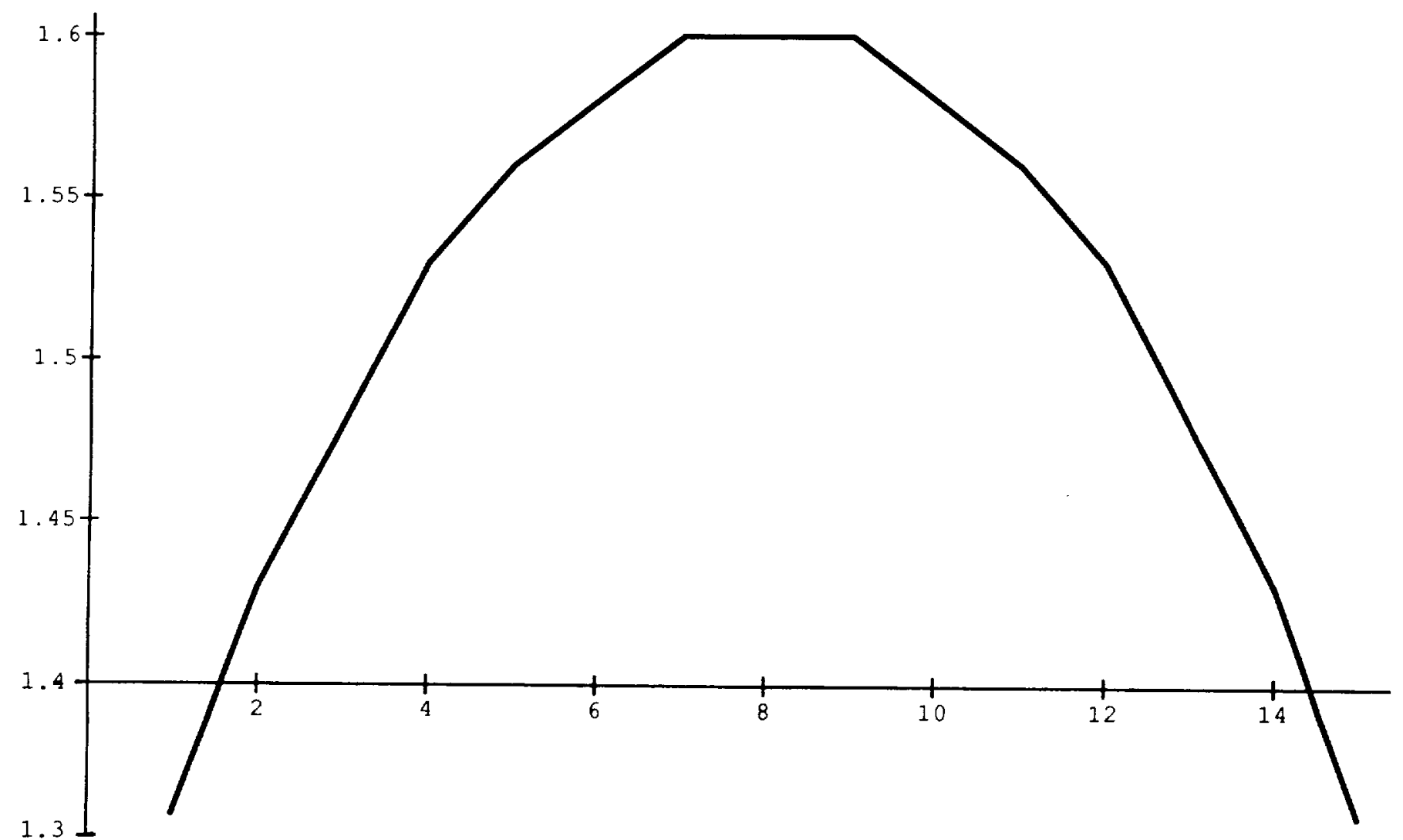

FIGURE 6

MIDACTUATOR POSITION: INCREMENTS L/16 


\section{Optimal Location of Controllers}

As a byproduct of our theory, we can examine the problem of the optimal actuators placement that minimizes the mean square attitude for any given choice of control gains and structure parameters.

\section{Case 1: One Actuator}

Here we take the criterion as the sum of the mean square displacements at both ends. We calculate explicitly that

$$
\begin{aligned}
& \frac{\left[E\left[f(0, t) f(0, t)^{*}\right]+E\left[f(L, t) f(L, t)^{*}\right]\right]}{d} \\
& =\text { Diag. }\left[\frac{2}{\alpha}, \frac{2+s^{2}+(L-s)^{2}}{\alpha}, \frac{2+s^{2}+(L-s)^{2}}{\alpha}, \frac{2}{\alpha}, \frac{2}{\alpha}, \frac{2}{\alpha}\right]
\end{aligned}
$$

from which it is clear that the optimal placement is in the middle:

$$
s=\frac{L}{2} \text {. }
$$

Only the bending-displacement is affected by actuator position.

For Case 2 of two actuators there is no placement problem since one is required to be at each end.

\section{Case 3: Three Actuators}

Here we can consider the optimal placement of the midactuator while the other two are fixed one at each end. The behavior of the sum of the mean square errors (setting $\alpha=1$ ) at both ends for the axial mode is shown in Figure 3; and the bending mode is shown in Figure 4; and the axial torsion in Figure 5. In all cases we see that the worst position is at the middle! The best place is at either end. Finally Figure 6 shows the mean square axial displacement at the actuator location. Again the worst place is the middle.

\section{4}




\section{Optimization}

We shall now treat a canonical optimization problem currently studied by FEM and truncated modal models $[9,10]$. The objective is to minimize the total mass - structure and control - subject to meeting specified indices of performance. Here we take them to be:

(a) mean square attitude error due to sensor noise less than or equal to fixed value

(b) stability index: sum of the absolute values of the real parts of the closed loop eigenvalues to be not less than a fixed value.

We shall see that the problem can be solved analytically by virtue of the formulas we have developed using continuum models.

The structural parameter we shall use is the cross-sectional area $A$ of the longerons (assumed to be the same for battens and cross-bars). Other parameters being fixed, the structure mass is then proportional to $A$. (The extension to the case of nonequal areas only complicates the algebra, as can be seen from the expressions (cf. Section 3 ) for the flexibility coefficients.) The control mass has to be subdivided into a stationary mass (armature mass, for example) and a moving mass (rotor mass, for example) since only the latter is involved in the stability index formula. The stationary mass is of course related to the moving mass - for simplicity we shall take it to be inversely proportional to the rotor mass. The control parameters are the attiude and rate gains $\alpha$ and $\gamma$. These of course will need to be constrained not to exceed prescribed limits. Thus we have the following formulation (nominal values for all structure parameters except $A$ ):

$$
\begin{aligned}
& \text { Structure mass }=N \ell \rho A \\
& \text { Control stationary mass }=\frac{k}{m} \\
& \text { Moving mass }=m \\
& \text { Total mass }=N \ell \rho A+\frac{k}{m}+m \\
& \text { Stability index }=\frac{\gamma}{M+m} \\
& 285
\end{aligned}
$$


(where $M$ denotes the contribution of the end-masses). For the truss considered,

$$
N=(76+46 \sqrt{2})
$$

Finally the mean square attitude error - to be specific, we shall consider the case of two controllers, one at each end; and take the sum of the mean square displacements at either end. First we express these in terms of the structural parameter $A$ - we have thus to use the expressions we have derived for the flexibility coefficients $\left\{c_{i j}\right\}$ in terms of $A$ in Section 5 and substitute them into the formulas for mean square errors for two controllers in Table 3 under Case 2. In doing so we shall also take advantage of the simplification possible by noting that for the nominal value of $L=27$ meters, we can readily calculate that

$$
\lambda \beta \geq 2
$$

so that we may replace both $(\lambda \beta-1)$ and $(\lambda \beta-2)$ by $\lambda \beta$. Thus the first four diagonal terms in

2 Diag. $E\left[f(0, t) f(0, t)^{*}\right]$

$$
\left(=2 \text { Diag. } E\left[f(L, t) f(L, t)^{*}\right]\right)
$$

are given in order by:

$$
\begin{aligned}
& f(\alpha, \gamma, A)=\frac{1}{\alpha}\left[1+\frac{\frac{L^{2}}{4}+\frac{L}{2 c \delta} \frac{\alpha}{E A}}{2+\frac{L^{2}}{4}+\frac{L}{2 c \delta} \frac{\alpha}{E A}+\left(\frac{c_{11}-2 c}{2 c \delta}\right)\left(\frac{2 \alpha+L c \delta E A}{\alpha}\right)}\right] d \\
& \frac{1}{\alpha}\left[1+\frac{\frac{L^{2}}{4}+\frac{L}{2 c \delta} \frac{\alpha}{E A}}{1+\frac{L^{2}}{4}+\frac{L}{2 c \delta} \frac{\alpha}{E A}}\right] d \\
& \frac{1}{\alpha}\left[1+\frac{\frac{L^{2}}{4}+\frac{L}{2 c \delta} \frac{\alpha}{E A}}{1+\frac{L^{2}}{4}+\frac{L}{2 c \delta} \frac{\alpha}{E A}}\right] d \\
& {\left[\frac{1}{\alpha}+\frac{L}{2 E A} \frac{c_{33}+\frac{c_{66}}{4}+\frac{L}{2} \frac{\alpha}{E A}}{\left(c_{33}+\frac{c_{66}}{4}+\frac{L}{2} \frac{\alpha}{E A}\right)\left(c_{66}+\frac{L}{2} \frac{\alpha}{E A}\right)-\frac{c_{66}^{2}}{2}}\right] d} \\
& 286
\end{aligned}
$$


where now

$$
\begin{aligned}
c_{11} & =\frac{40+24 \sqrt{2}}{9+4 \sqrt{2}} \\
c & =\frac{2}{1+2 \sqrt{2}} \\
c_{33} & =n\left(\frac{2725+1476 \sqrt{2}}{2628+1336 \sqrt{2}}\right) \\
c_{66} & =n\left(\frac{32+66 \sqrt{2}}{296+130 \sqrt{2}}\right) \\
n & =\text { number of bays } \quad L=n \ell \\
\delta & =\frac{1}{1+\frac{L^{2}}{12} \cdot \frac{c}{\left(c_{33}-c_{66} / 4\right)}} .
\end{aligned}
$$

with $d$ as given by (5.7). These formulas enable us to draw conclusions concerning the dependence on the cross-sectional area $A$ without resorting to numerical computer calculations. We see that all the errors decrease as $A$ increases. The axial error decreases from $\frac{2 d}{\alpha}$ at $A=0$ to $\frac{d}{\alpha}$ at $A=\infty$; similarly the torsion error. The bending error is least affected, decreasing from $\frac{d}{\alpha}$ at $A=0$ to $\left(\frac{L^{2}}{4+L^{2}}\right) \frac{d}{\alpha}$ at $A=\infty$. In all cases the minimal mean square error is at most $3 \mathrm{db}$ less than the maximum!

For the optimization let us fix on the mean square bending error as being the easiest analytically: let

$$
f(\alpha, \gamma, A)=\frac{d}{\alpha}\left[1-\frac{1}{1+\frac{L^{2}}{4}+\frac{L}{2 c \delta} \frac{\alpha}{E A}}\right]
$$

Thus the optimization problem is that of minimizing

$$
N \ell \rho A+\frac{k}{m}+m
$$

subject to:

$$
\begin{gathered}
\frac{\gamma}{M+m} \geq \sigma_{s}^{2} \\
f(\alpha, \gamma, A) \leq \sigma_{a}^{2} .
\end{gathered}
$$


The first inequality can clearly be reversed to read

$$
\frac{M+m}{\gamma} \leq \frac{1}{\sigma_{s}^{2}}
$$

We note that the objective functional

$$
N \ell p A+\frac{k}{m}+m
$$

is infinitely smooth and trivially convex, and the constraints are also infinitely smooth and convex. Hence we are assured of the existence of a minimum (which is further verified to be unique). Moreover we can go to the Langrange parameter formulation and minimize:

$$
\left(N \ell \rho A+\frac{k}{m}+m\right)+\lambda_{1}\left(\frac{M+m}{\gamma}\right)+\lambda_{2} f(\alpha, \gamma, A)
$$

where $\lambda_{1}, \lambda_{2} \geq 0$ are the Lagrange parameters. See [11] for the standard results that are applicable here.

Compared to the FEM versions $[9,10]$ this is a "trivial" problem and complete "analytical" solution is possible. We omit the details since our primary aim in this paper is to demonstrate the simplicity of the optimization problem relative to the FEM versions.

\section{Acknowledgement}

Grateful acknowledgement is made of several informal discussions with E. Armstrong and S. Joshi of the CSI Analytical Design Methods Team at NASA Langley FRC on the problem; and of the FEM calculations by H-C. Wang. 


\section{References}

1. A. K. Noor and W. C. Russell. "Anisotropic Continuum Models for Beamlike Lattice Trusses." Computer Methods in Applied Mechanics and Engineering, 1986, pp. 257-277.

2. A. K. Noor and C. M. Andersen. "Analysis of Beamlike Trusses." Computer Methods in Applied Mechanics and Engineering, 1978, pp. 53-70.

3. J. D. Renton. "The Beam-like Behavior of Space Trusses." AIAA Journal, Vol. 22, No. 2 (Feb 1984).

4. A. V. Balakrishnan. "Feedback Control for Stabilization of Flexible Multibody Structures." In: Differential Equations: Stability and Control. New York: Marcel Dekker, 1990.

5. L. W. Taylor. "Distributed Parameter Modeling for the Control of Flexible Spacecraft." In: Proceedings of the NASA-UCLA Workshop on Computational Techniques in Identification and Control of Flexible Flight Structures, Lake Arrowhead, November 1989. New York-Los Angeles: OSI Publications, 1990. Pp. 87-114.

6. A. V. Balakrishnan. "Combined Structures-Controls-Integrated Optimization Using Distributed Parameter Models." In: Proceedings of the NASA-UCLA Workshop on Computational Techniques in Identification and Control of Flexible Flight Structures, Lake Arrowhead, November 1989. OSI Publications, 1990. Pp. 70-86.

7. A. V. Balakrishnan. "Dynamics of Anisotropic Timoshenko Beams with Distributed Controls." To appear.

8. A. V. Balakrishnan. "A Mathematical Formulation of the SCOLE Control Problem, Part II: Optimal Compensator Design." NASA CR 181720. December 1988.

9. M. Salama, J. Garha, L. Demsetz and F. Udwadia. "Simultaneous Optimization of Control Structures." Computational Mechanics," Vol. 3 (1988), pp. 257-282.

10. Sandeep Gupta and S. M. Joshi. "An Integrated Control/Structure Design Method Using Multiobjective Optimization." Presented at the 4th NASA Workshop on Computational Control of Flexible Aerospace Systems, Williamsburg, Virginia, July 1990. Preprint.

11. B. T. Polyak. Introduction to Optimization. OSI Publications, 1987.

end file: july 90 